Supplement of Hydrol. Earth Syst. Sci., 23, 3765-3786, 2019

https://doi.org/10.5194/hess-23-3765-2019-supplement

(c) Author(s) 2019. This work is distributed under

the Creative Commons Attribution 4.0 License.

(c) (1)

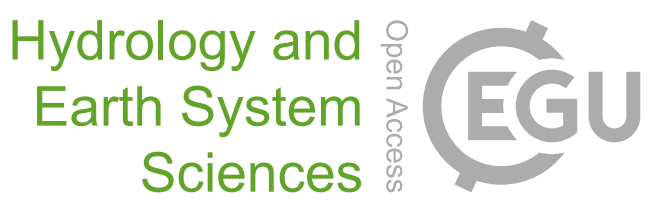

Supplement of

\title{
The sensitivity of modeled snow accumulation and melt to precipitation phase methods across a climatic gradient
}

Keith S. Jennings and Noah P. Molotch

Correspondence to: Keith S. Jennings (keithj@unr.edu)

The copyright of individual parts of the supplement might differ from the CC BY 4.0 License. 
The following plots (Fig. S1-S11) show mean daily SWE at the 11 study stations using the 12 different precipitation phase methods as denoted by the colored lines. The mean daily SWE was computed by averaging the SWE on each day for the given precipitation phase method across the simulation years.

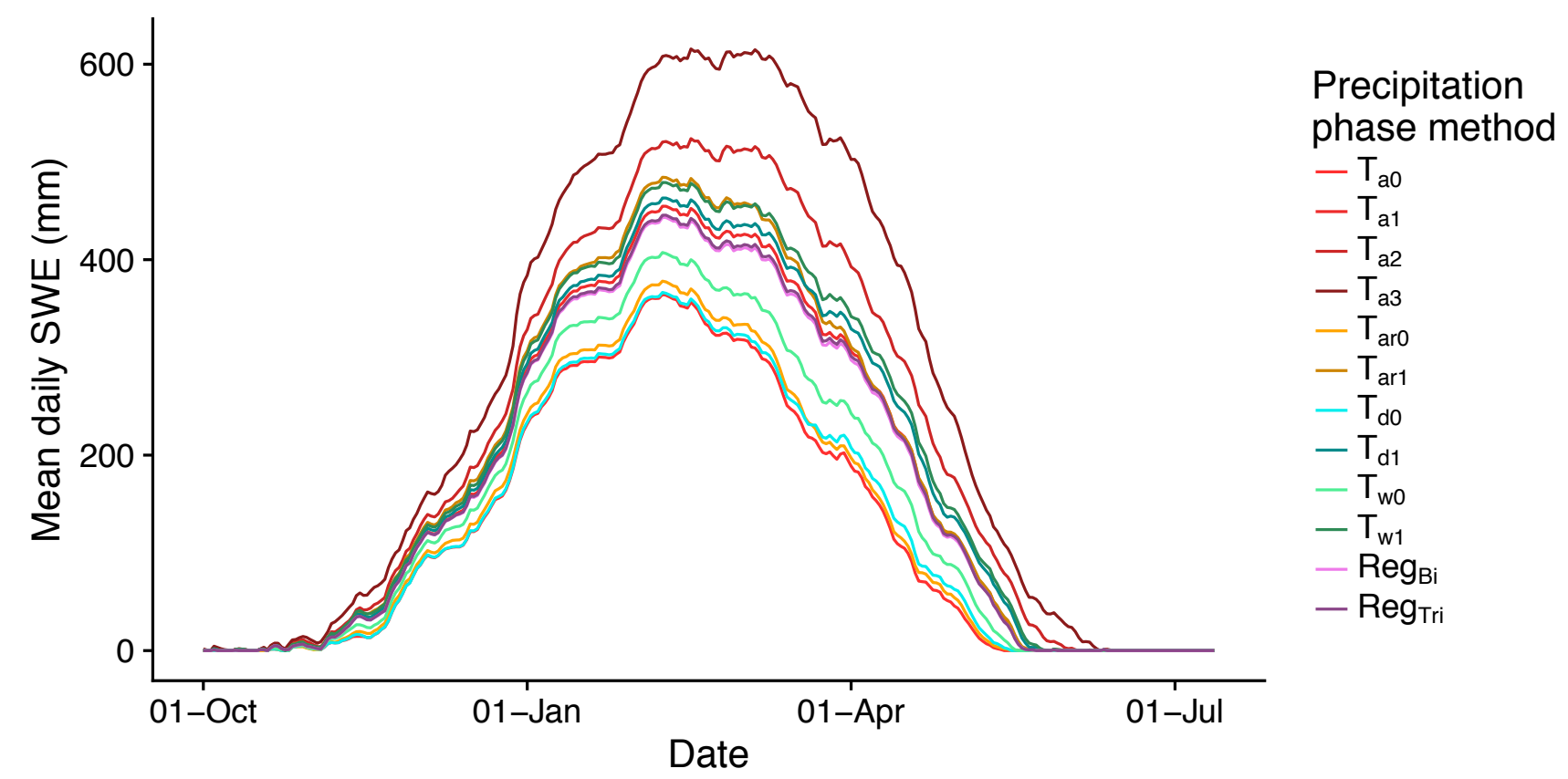

5 Figure S1. Mean daily SWE for the different precipitation phase methods at HJA-CEN. 


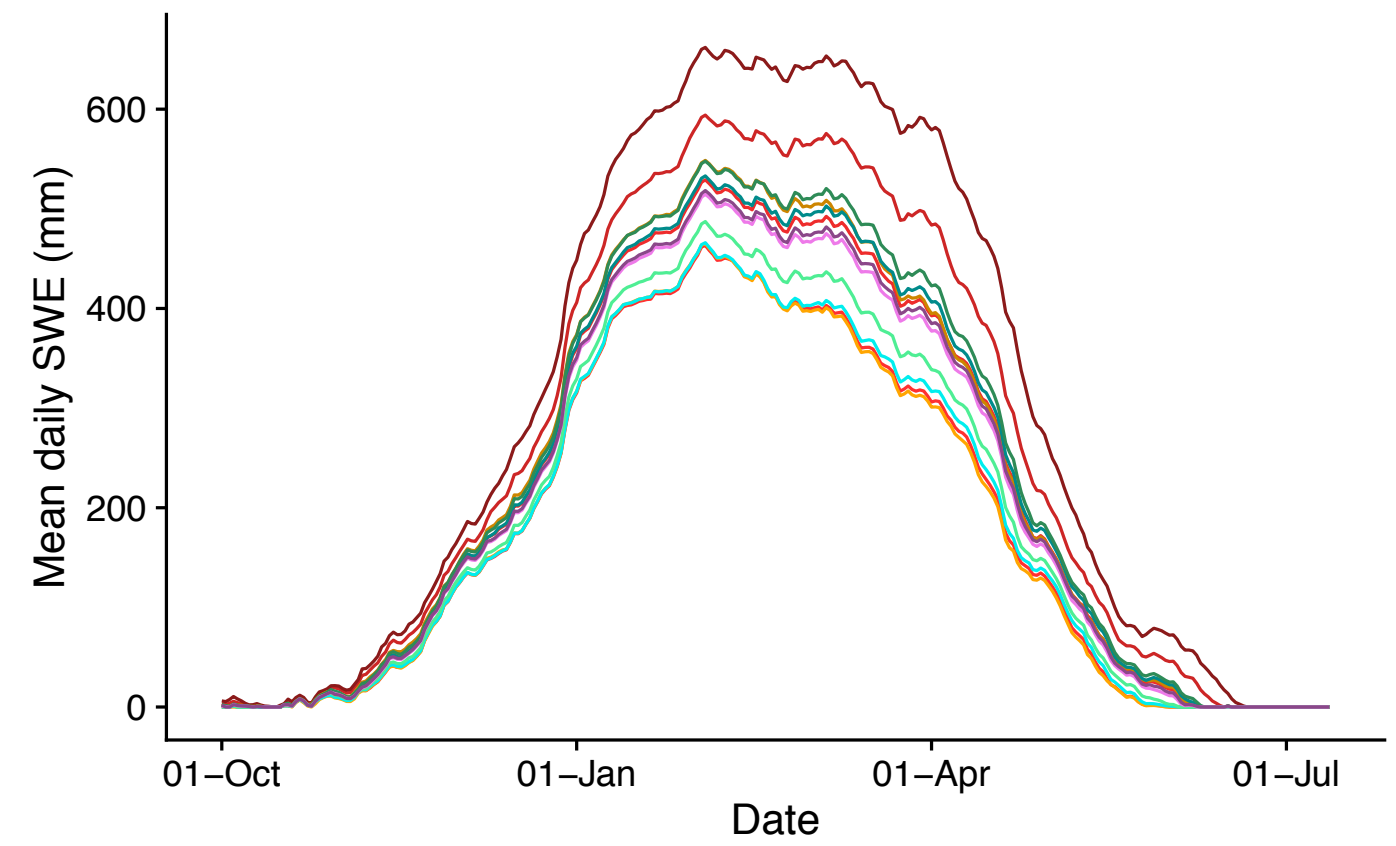

Precipitation phase method

$-\mathrm{T}_{\mathrm{a} 0}$

$-\mathrm{T}_{\mathrm{a} 1}$

$-\mathrm{T}_{\mathrm{a} 2}$

$-\mathrm{T}_{\mathrm{a} 3}$

- $\mathrm{T}_{\mathrm{aro}}$

$-\mathrm{T}_{\mathrm{ar} 1}$

$-\mathrm{T}_{\mathrm{d} 0}$

$-T_{\mathrm{d} 1}$

$-\mathrm{T}_{\mathrm{w0}}$

$-\mathrm{T}_{\mathrm{w} 1}$

- Reg $_{\mathrm{Bi}}$

- Reg $_{T r i}$

Figure S2. Mean daily SWE for the different precipitation phase methods at HJA-VAN.

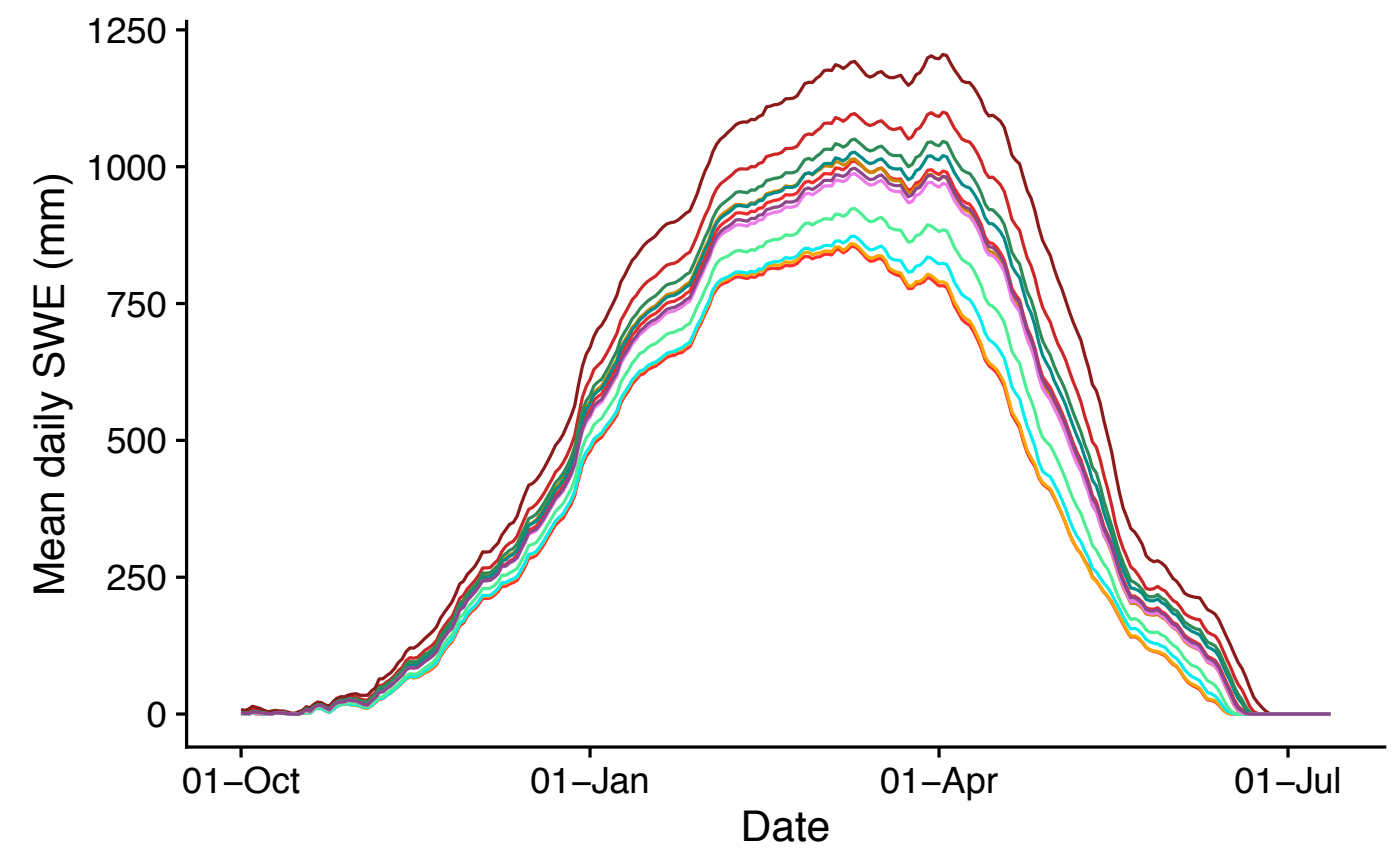

Precipitation phase method

$-\mathrm{T}_{\mathrm{a} 0}$

$-\mathrm{T}_{\mathrm{a} 1}$

$-\mathrm{T}_{\mathrm{a} 2}$

$-\mathrm{T}_{\mathrm{a} 3}$

$-\mathrm{T}_{\text {aro }}$

$-\mathrm{T}_{\mathrm{ar} 1}$

$-T_{\mathrm{d} 0}$

$-\mathrm{T}_{\mathrm{d} 1}$

$-T_{w 0}$

$-\mathrm{T}_{\mathrm{w} 1}$

- Reg $_{\mathrm{Bi}}$

- Reg $_{T r i}$

5

Figure S3. Mean daily SWE for the different precipitation phase methods at HJA-UPL. 


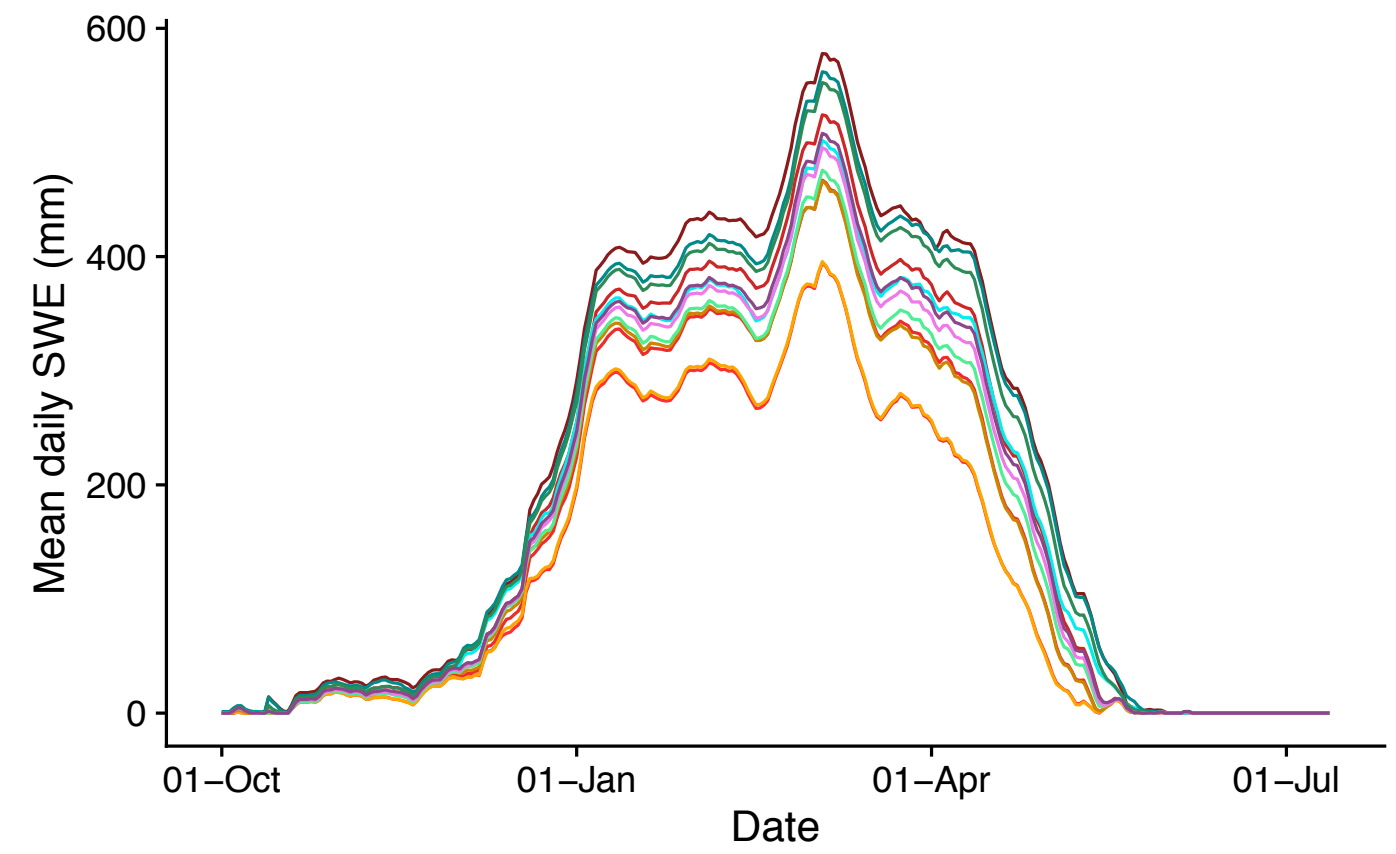

Precipitation phase method

$-\mathrm{T}_{\mathrm{a} 0}$

$-\mathrm{T}_{\mathrm{a} 1}$

$-\mathrm{T}_{\mathrm{a} 2}$

$-\mathrm{T}_{\mathrm{a} 3}$

- $\mathrm{T}_{\text {aro }}$

$-\mathrm{T}_{\mathrm{ar} 1}$

$-\mathrm{T}_{\mathrm{d} 0}$

$-T_{\mathrm{d} 1}$

$-T_{w 0}$

$-T_{w 1}$

- Reg $_{\mathrm{Bi}}$

- Reg $_{T r i}$

Figure S4. Mean daily SWE for the different precipitation phase methods at SSC-LWR.

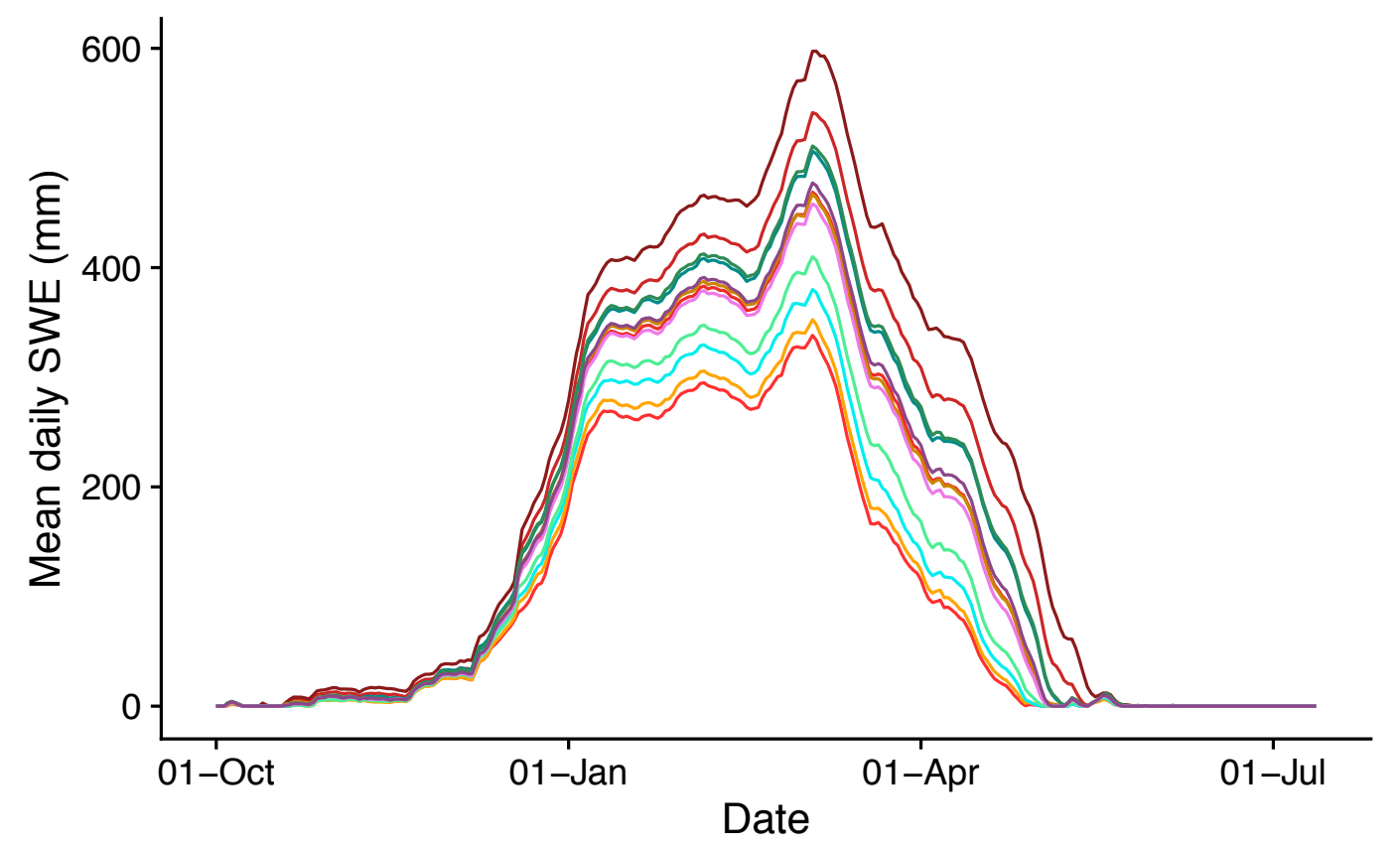

Precipitation phase method

$-\mathrm{T}_{\mathrm{a} 0}$

$-\mathrm{T}_{\mathrm{a} 1}$

$-\mathrm{T}_{\mathrm{a} 2}$

$-\mathrm{T}_{\mathrm{a} 3}$

- $\mathrm{T}_{\mathrm{ar} 0}$

$-T_{\text {ar1 }}$

$-\mathrm{T}_{\mathrm{d} 0}$

$-T_{\mathrm{d} 1}$

$-T_{\text {wo }}$

$-T_{w 1}$

- Reg $_{\mathrm{Bi}}$

- Reg $_{T r i}$

5 Figure S5. Mean daily SWE for the different precipitation phase methods at SSC-UPR. 


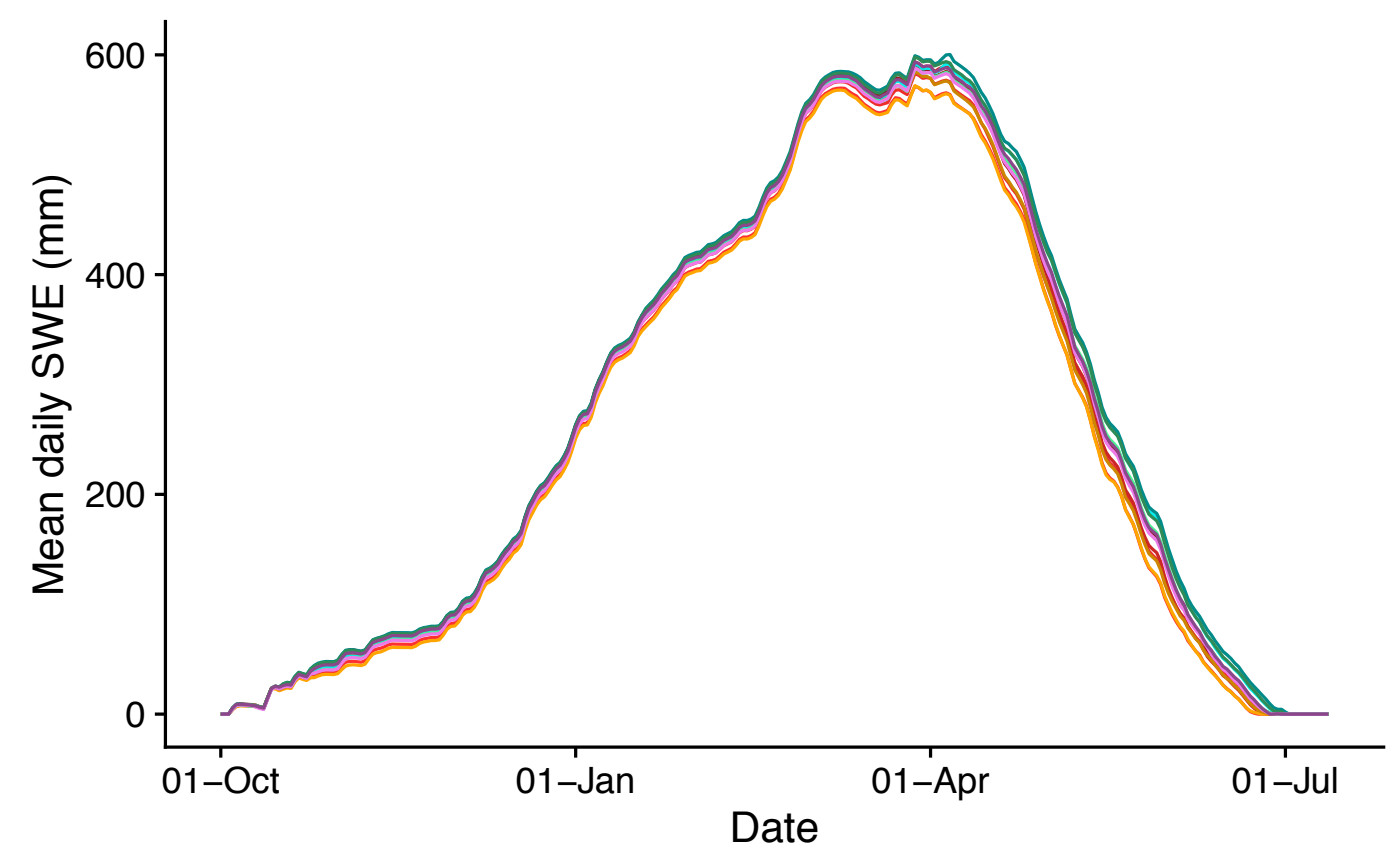

Precipitation phase method

$-\mathrm{T}_{\mathrm{a} 0}$

$-\mathrm{T}_{\mathrm{a} 1}$

$-\mathrm{T}_{\mathrm{a} 2}$

$-\mathrm{T}_{\mathrm{a} 3}$

- $\mathrm{T}_{\text {aro }}$

$-\mathrm{T}_{\mathrm{ar} 1}$

$-\mathrm{T}_{\mathrm{d} 0}$

$-T_{d 1}$

$-T_{w 0}$

$-T_{\mathrm{w} 1}$

- Reg $_{\mathrm{Bi}}$

- Reg $_{T r i}$

Figure S6. Mean daily SWE for the different precipitation phase methods at YOS-DAN.

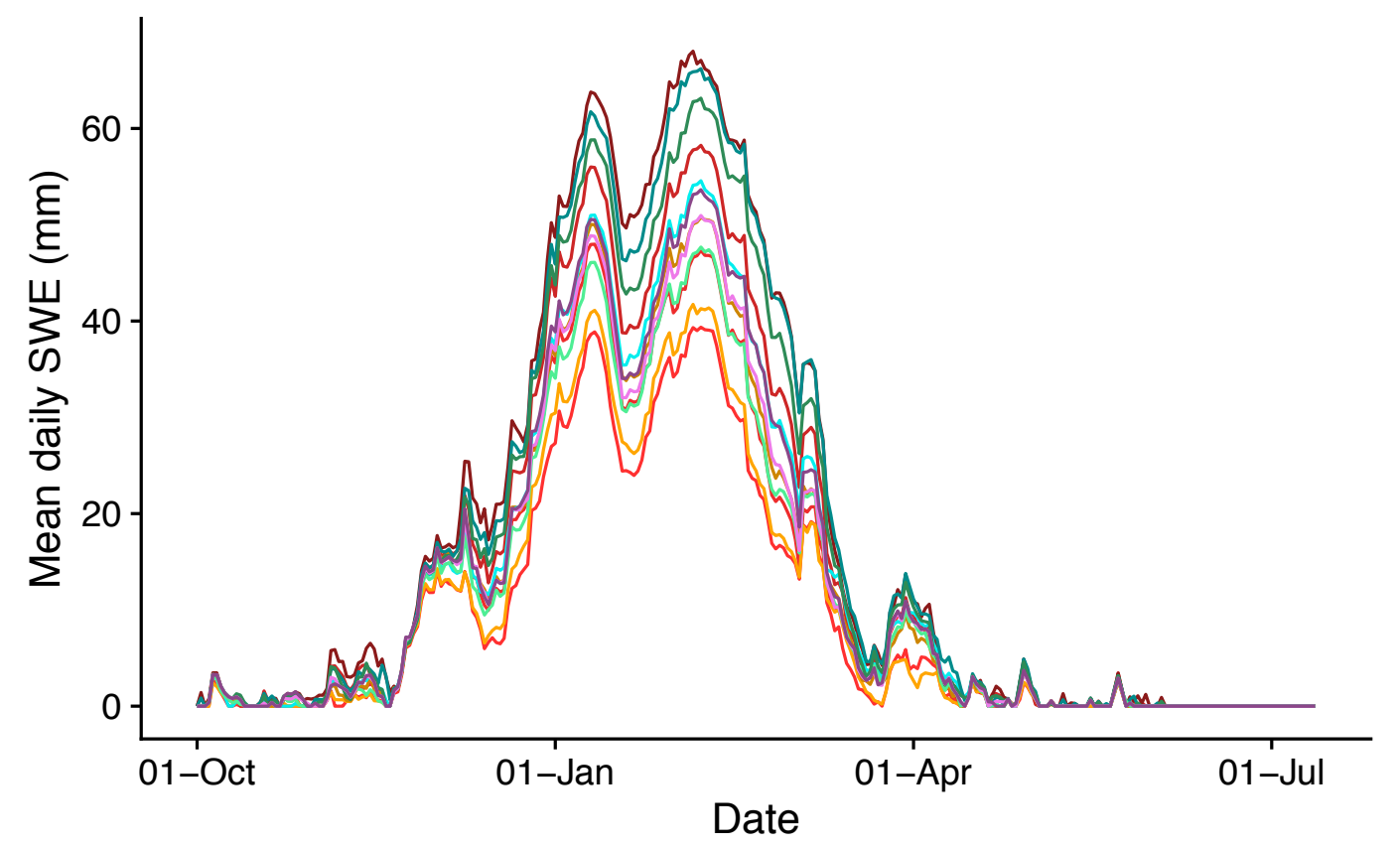

Precipitation phase method

$-\mathrm{T}_{\mathrm{a} 0}$

$-\mathrm{T}_{\mathrm{a} 1}$

$-\mathrm{T}_{\mathrm{a} 2}$

$-\mathrm{T}_{\mathrm{a} 3}$

- $\mathrm{T}_{\mathrm{ar} 0}$

$-T_{\text {ar1 }}$

$-\mathrm{T}_{\mathrm{d} 0}$

$-T_{\mathrm{d} 1}$

$-T_{\text {wo }}$

$-T_{w 1}$

- Reg $_{\mathrm{Bi}}$

- Reg $_{T r i}$

5 Figure S7. Mean daily SWE for the different precipitation phase methods at JD-125. 


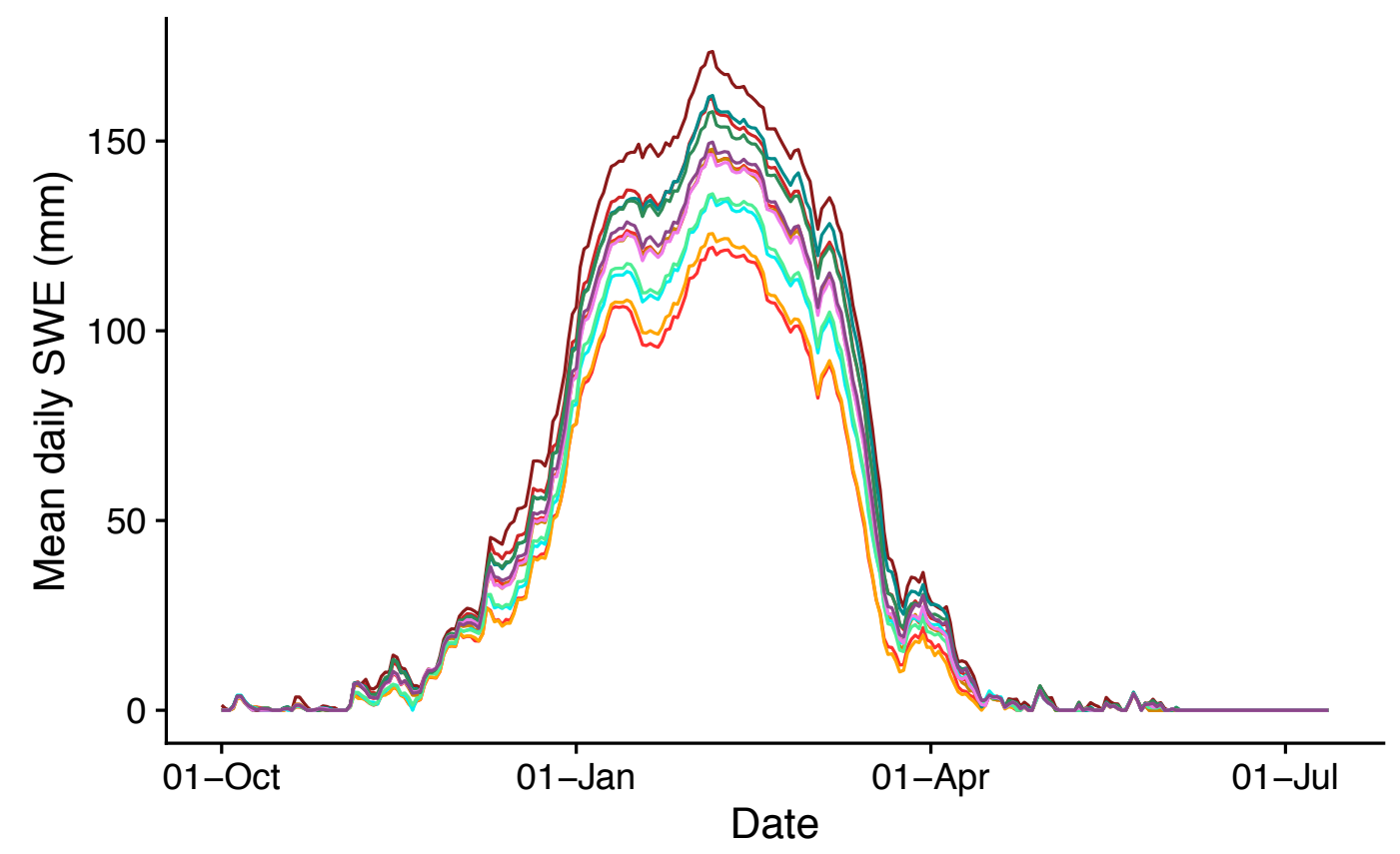

Precipitation phase method

$-\mathrm{T}_{\mathrm{a} 0}$

$-\mathrm{T}_{\mathrm{a} 1}$

$-\mathrm{T}_{\mathrm{a} 2}$

$-\mathrm{T}_{\mathrm{a} 3}$

- $\mathrm{T}_{\text {aro }}$

$-\mathrm{T}_{\mathrm{ar} 1}$

$-\mathrm{T}_{\mathrm{d} 0}$

$-T_{d 1}$

$-T_{w 0}$

$-T_{w 1}$

- Reg $_{\mathrm{Bi}}$

- Reg $_{T r i}$

Figure S8. Mean daily SWE for the different precipitation phase methods at JD-124b.

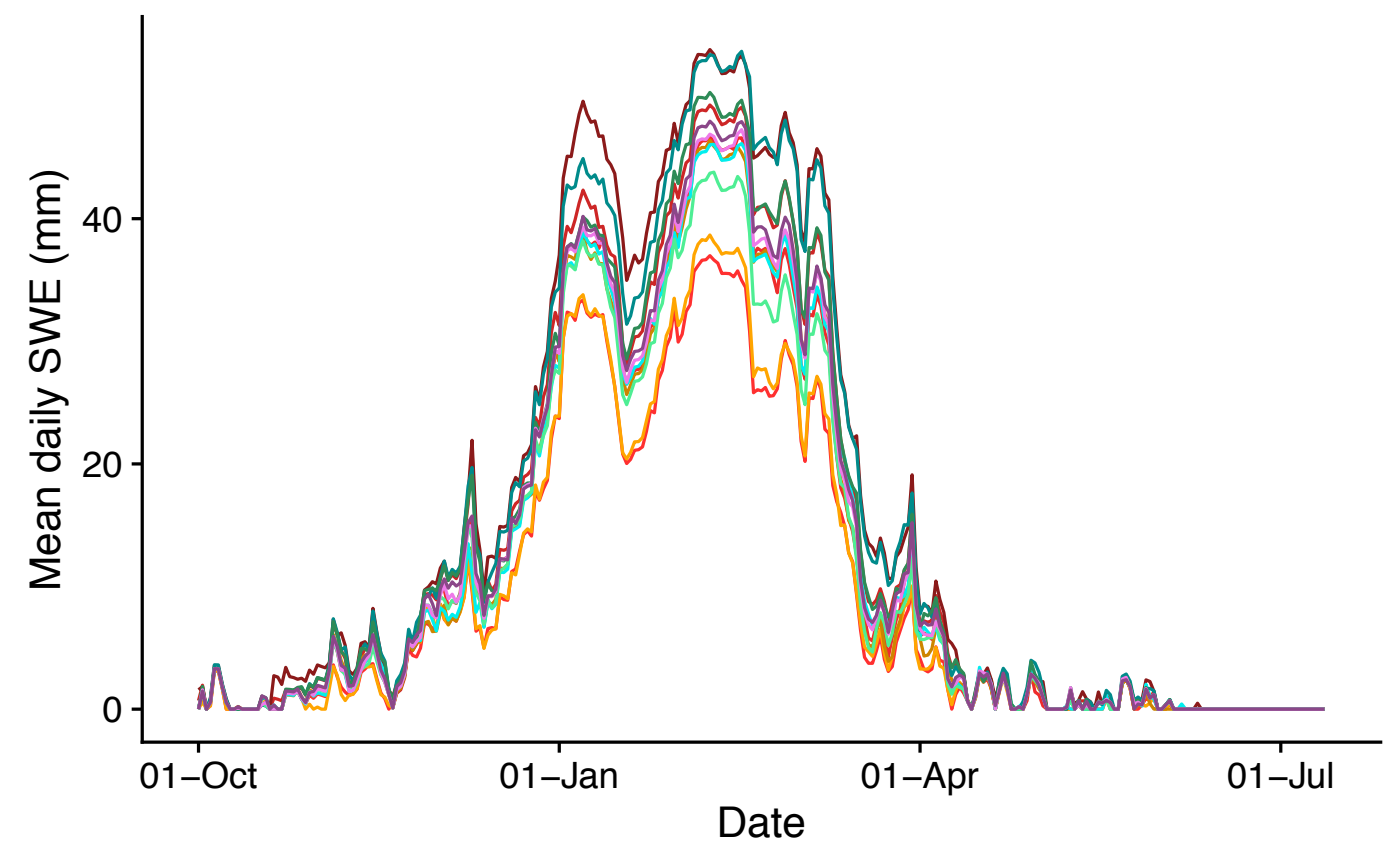

Precipitation phase method

$-\mathrm{T}_{\mathrm{a} 0}$

$-\mathrm{T}_{\mathrm{a} 1}$

$-\mathrm{T}_{\mathrm{a} 2}$

$-\mathrm{T}_{\mathrm{a} 3}$

- $\mathrm{T}_{\mathrm{ar} 0}$

$-T_{\text {ar1 }}$

$-\mathrm{T}_{\mathrm{d} 0}$

$-T_{\mathrm{d} 1}$

$-T_{\text {wo }}$

$-T_{w 1}$

- Reg $_{\mathrm{Bi}}$

- Reg $_{T r i}$

5 Figure S9. Mean daily SWE for the different precipitation phase methods at JD-124. 


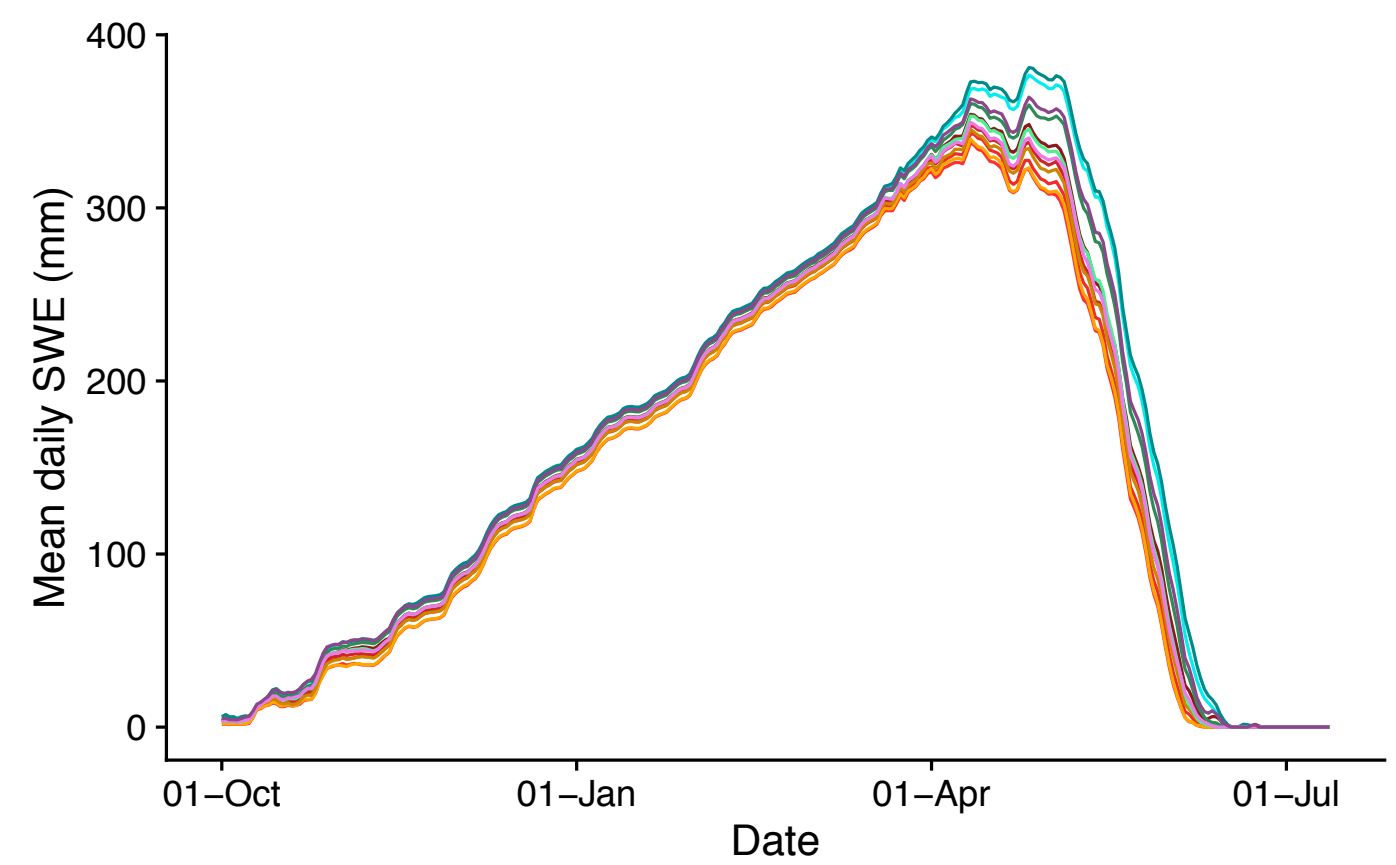

Precipitation phase method

$-\mathrm{T}_{\mathrm{a} 0}$

$-\mathrm{T}_{\mathrm{a} 1}$

$-\mathrm{T}_{\mathrm{a} 2}$

$-\mathrm{T}_{\mathrm{a} 3}$

- $\mathrm{T}_{\text {aro }}$

$-\mathrm{T}_{\mathrm{ar} 1}$

$-\mathrm{T}_{\mathrm{d} 0}$

$-T_{d 1}$

$-T_{\text {wo }}$

$-T_{w 1}$

- Reg $_{\mathrm{Bi}}$

- Reg $_{T r i}$

Figure S10. Mean daily SWE for the different precipitation phase methods at NWT-C1.

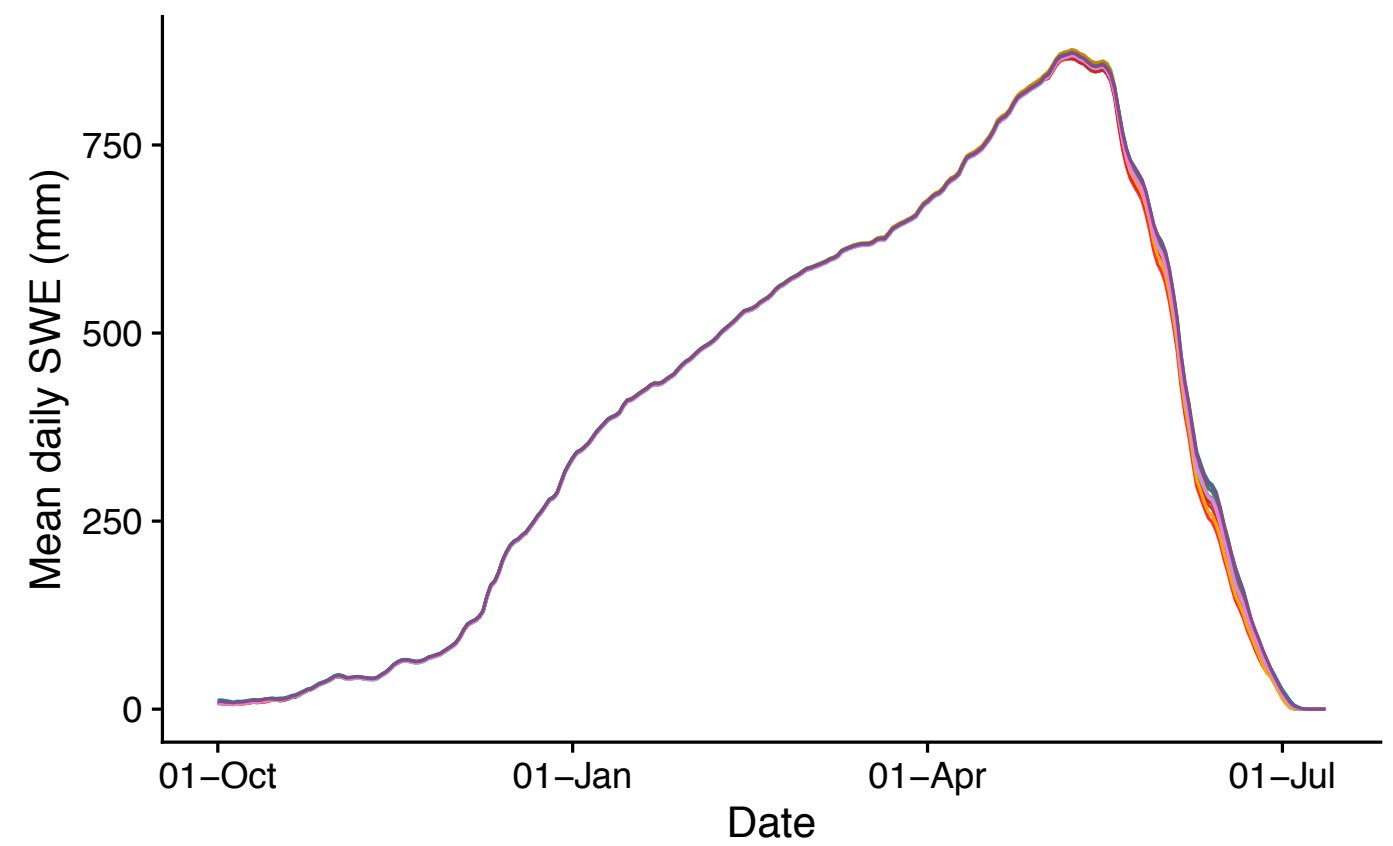

Precipitation phase method

$-\mathrm{T}_{\mathrm{a} 0}$

$-\mathrm{T}_{\mathrm{a} 1}$

$-\mathrm{T}_{\mathrm{a} 2}$

$-\mathrm{T}_{\mathrm{a} 3}$

- $\mathrm{T}_{\mathrm{ar} 0}$

$-T_{\text {ar1 }}$

$-T_{\mathrm{d} 0}$

$-T_{\mathrm{d} 1}$

$-T_{\text {wo }}$

$-T_{w 1}$

- Reg $_{\mathrm{Bi}}$

- Reg Tri

5 Figure S11. Mean daily SWE for the different precipitation phase methods at NWT-SDL. 
Table S1. Measurement heights for wind speed and other meteorological quantities at the study stations.

\begin{tabular}{lcc} 
Station & $\begin{array}{c}\text { Wind height } \\
(\mathrm{m})\end{array}$ & $\begin{array}{c}\text { Other measurement } \\
\text { heights }(\mathrm{m})\end{array}$ \\
\hline HJA-CEN & 10 & 4.5 \\
HJA-VAN & 10 & 4.5 \\
HJA-UPL & 10 & 4.5 \\
SSC-LWR & 4 & 4 \\
SSC-UPR & 4 & 4 \\
YOS-DAN & 5 & 5 \\
JD-125 & 3 & 3 \\
JD-124b & 3 & 3 \\
JD-124 & 3 & 3 \\
NWT-C1 & $5 *$ & 2 \\
NWT-SDL & 5 & 2
\end{tabular}

*The NWT-C1 anemometer is located in an unrepresentative location (open road), so observations were corrected to represent wind speed in the forest canopy (Jennings et al., 2018a).

5 Table S2. Optimized rain-snow air temperature thresholds for each station in the study using four different data sources: 1-Map) The spatially continuous threshold map from Jennings et al. (2018); 2-Obs.) The observed threshold from the closest meteorological station (Jennings et al., 2018); 3-SWE) The threshold inferred from changes in SWE (Fig. S12); and 4-Depth) The threshold inferred from changes in snow depth at each study station (Fig. S13). An NA indicates there were insufficient data to estimate the threshold from SWE and/or snow depth. The Mean column gives the optimized threshold for each station as the

10 arithmetic mean of all valid thresholds, while the Site mean is the arithmetic mean of the Mean column for each site, rounded to the nearest integer value to be consistent with the other thresholds in this work.

\begin{tabular}{lcccccc}
\multicolumn{7}{c}{ Optimized rain-snow air temperature threshold $\left({ }^{\circ} \mathrm{C}\right)$} \\
Station & Map & Obs. & SWE & Depth & Mean & Site mean \\
\hline HJA-CEN & 1.19 & 1.12 & 1.53 & -0.27 & 0.89 & 1.0 \\
HJA-VAN & 1.19 & 1.12 & 1.45 & -0.2 & 0.89 & 1.0 \\
HJA-UPL & 1.19 & 1.12 & 1.96 & -0.36 & 0.98 & 1.0 \\
SSC-LWR & 1.7 & $1^{*}$ & NA & 1.34 & 1.35 & 1.0 \\
SSC-UPR & 1.7 & $1^{*}$ & 1.81 & 0.78 & 1.32 & 1.0 \\
YOS-DAN & 2.21 & 2.78 & NA & NA & 2.50 & 2.0 \\
JD-125 & 2.25 & 1.25 & NA & 2.4 & 1.97 & 2.0 \\
JD-124b & 2.25 & 1.25 & NA & 0.95 & 1.48 & 2.0 \\
JD-124 & 2.25 & 1.25 & NA & 1.04 & 1.51 & 2.0 \\
NWT-C1 & 2.84 & 2.34 & 3.57 & NA & 2.92 & 3.0 \\
NWT-SDL & 2.84 & 2.34 & NA & NA & 2.59 & 3.0
\end{tabular}

*The Obs. threshold for SSC was taken from the second closest precipitation phase observation station because the closest was located on the east side of the Sierra, which has a markedly different climate. 


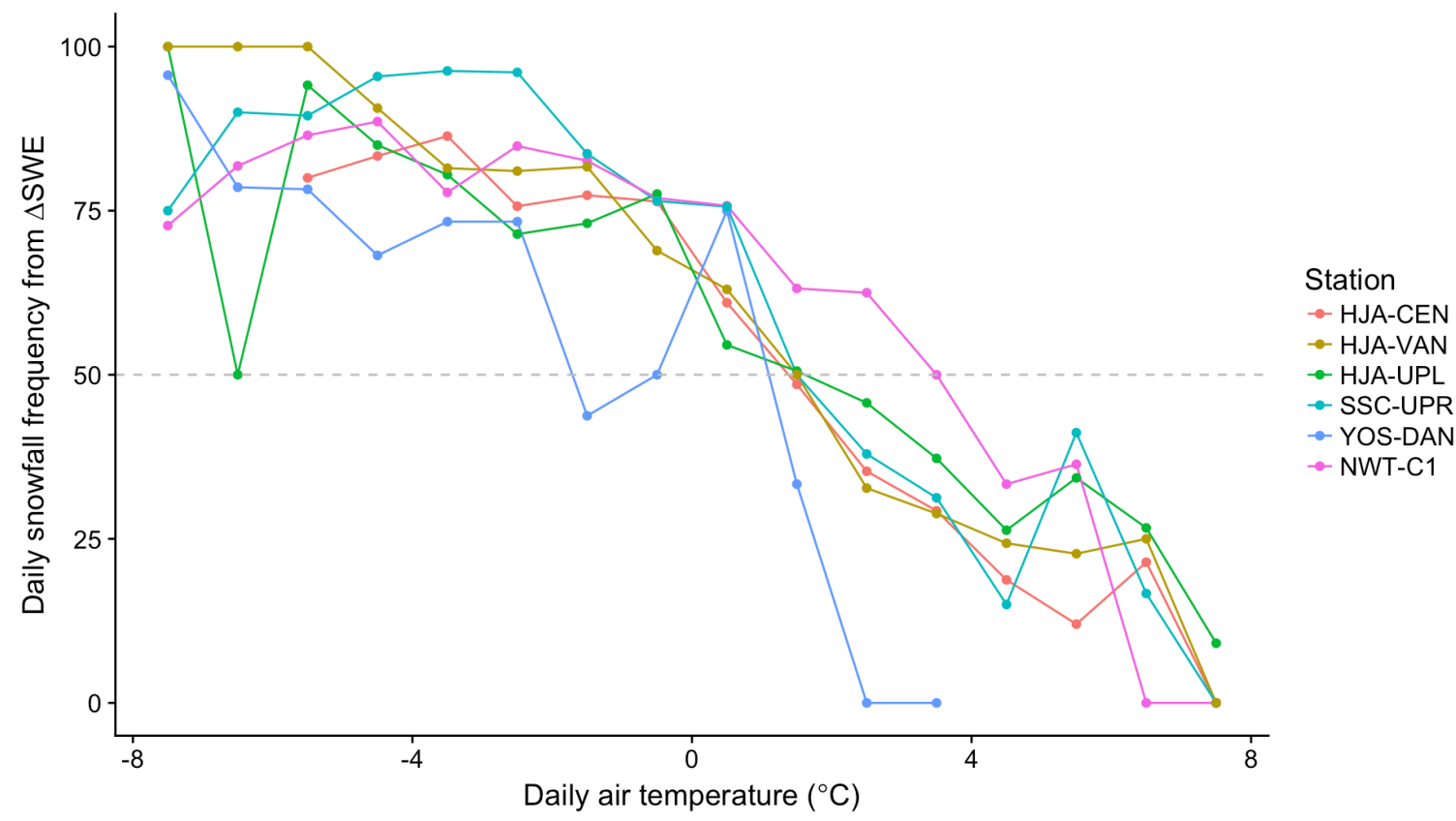

Figure S12. Snowfall frequency per $1^{\circ} \mathrm{C}$ air temperature bin as computed from SWE data. On days with precipitation $>2.54$ mm and SWE $>0 \mathrm{~mm}$, an increase in SWE was designated as snowfall, while no change or a decrease in SWE was designated as rainfall.

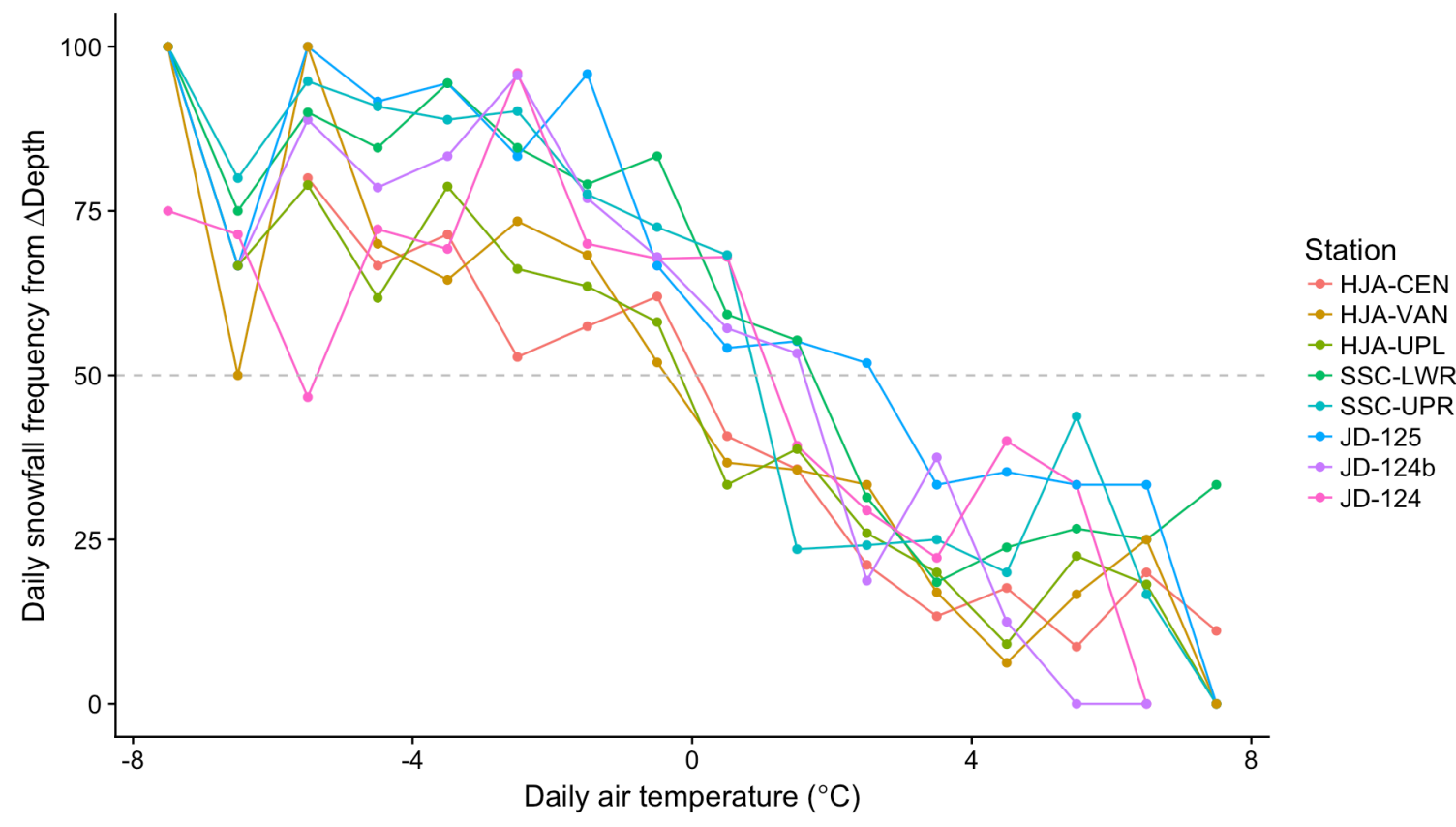

Figure S13. Snowfall frequency per $1^{\circ} \mathrm{C}$ air temperature bin as computed from snow depth data. On days with precipitation $>2.54$ $\mathrm{mm}$ and snow depth $>0 \mathrm{~mm}$, an increase in snow depth was designated as a snowfall event, while a zero change or decrease in snow depth was designated as rainfall. 